\title{
Single Incision Laparoscopic Transabdominal Preperitoneal Repair for Strangulated Groin Hernia
}

\author{
Po Ching Cathy Ng, George Pei Cheung Yang, Michael Ka Wah Li
}

Department of Surgery, Pamela Youde Nethersole Eastern Hospital, Hong Kong, China.

Email: cathay_pc@hotmail.com

Received March 11 ${ }^{\text {th }}, 2013$; revised May 5 ${ }^{\text {th }}, 2013$; accepted May 20 ${ }^{\text {th }}, 2013$

Copyright (C 2013 Po Ching Cathy Ng et al. This is an open access article distributed under the Creative Commons Attribution License, which permits unrestricted use, distribution, and reproduction in any medium, provided the original work is properly cited.

\begin{abstract}
Introduction: Single incision laparoscopic surgery (SILS) has become more popular for various surgical procedures including hernia surgery. Initial results of SILS in elective hernia repair were comparable to those of conventional laparoscopic approaches. However the use of SILS in emergency case has not been widely reported. This study aimed to evaluate the feasibility of the use of single incision laparoscopic transabdominal preperitoneal (TAPP) repair for patients presenting with strangulated groin hernia. Method: Emergency single incision laparoscopic TAPP repair were performed in our unit from June 2011 onwards for selected patients. Retrospectively data including the patient demographics, operative time, type of hernia, hospital stay, complications and recurrence rate were collected and analyzed. Result: There were a total of five patients in this series from June 2011 to June 2012. The median age was 62 years old with a male to female ratio of 4:1. Four patients had unilateral hernia (one femoral and three inguinal hernias) and one had bilateral hernia (unilaterally strangulated femoral hernia and bilaterally reducible indirect inguinal hernia). The median operative time was 75 minutes for patients with unilateral repair. None of the patients required bowel resection. The conversion rate to either conventional laparoscopic or open repair was zero. The median hospital stay was 2 days. No major complication or recurrence was detected. Conclusion: This series showed that single port laparoscopic TAPP repair for strangulated groin hernia is a feasible option with no major complication reported.
\end{abstract}

Keywords: Single Incision Laparoscopic Surgery; Transabdominal Preperitoneal Repair; Strangulated Hernia; Groin Hernia

\section{Introduction}

Groin hernia is a common surgical problem and was traditionally repaired with open technique. With the advance of laparoscopy, laparoscopic repair was shown to be comparable with open repair with lower wound infection rate and low recurrence rate in elective case [1]. Single incision transabdominal preperitoneal (TAPP) repair for groin hernia is another advancement of laparoscopic groin hernia surgery. The first single incision laparoscopic surgery (SILS) was performed in 2009 in our unit. The initial results of SILS in elective hernia repair in our center were comparable to those of conventional laparoscopic approaches [2].

Although laparoscopic groin hernia repair had been developed for more than 20 years, its application for acutely strangulated hernia was not widely accepted. Recent publication by Yang et al. in 2011 showed that laparoscopic repair for patients presenting with strangu- lated inguinal hernia was also feasible [3]. The use of SILS in emergency case has not been widely reported. This study aimed to evaluate the feasibility of use of single incision laparoscopic transabdominal preperitoneal (TAPP) repair for patients presented with acutely strangulated groin hernia.

\section{Method}

In our hospital, we first adapted the use of single incision TAPP repair for patients with strangulated inguinal hernia since June 2011. This was a retrospective study on a series of five patients presenting with strangulated inguinal hernia since June 2011 to June 2012 who received single incision laparoscopic hernia repair. Patients were selected if they have no previous abdominal operation, haemodynamically stable who can tolerate general anaesthesia, and with the availability of the specialist single incision hernia surgeon. The electronic patient record and 
the operation record were reviewed. The demographic data, perioperative parameters and post-operative outcomes were retrieved and analyzed.

\section{Technique}

Transumbilical single incision TAPP was performed under general anesthesia. The patient was placed in supine position. A Foley's catheter was inserted to decompress the urinary bladder preoperatively. The umbilicus was incised to create a $20 \mathrm{~mm}$ size wound for the insertion of SILS ${ }^{\mathrm{TM}}$ Port (Covidien, United States). The SILS ${ }^{\mathrm{TM}}$ Port is a flexible laparoscopic port that can accommodate up to three instruments through separate working channels. Carbon dioxide pneumoperitoneum was created at pressure of 10 to $12 \mathrm{mmHg}$. A $5 \mathrm{~mm} 30^{\circ}$ laparoscope (EndoEYE $^{\mathrm{TM}}$, Olympus, Japan) was used. The patient was placed in a Trendelenburg position. The content of the hernia sac was reduced mainly with external compression. Laparoscopic grasping instruments were used to aid the reduction of the hernia content only when necessary with caution not to damage the strangulated bowel loops. Any adhesions were taken down. The peritoneum over the side of the inguinal hernia was incised. Dissection of the preperitoneal space was performed. Any direct sac was reduced. Any indirect sac was ligated with endoties and transected distally. The spermatic cord was protected. Parietalization of the peritoneum was performed. A polyester mesh Parietex ADP2 (Covidien, United States) was placed to cover all myopectineal orifices. The peritoneal flap was closed with fixation device. The viability of the reduced content was reassessed again. Pneumoperitoneum was released after recheck laparoscopy. The umbilical wound was closed with PDS suture (PDS $^{\mathrm{TM}}$ II, Ethicon Inc., United States) and polypropylene suture subcuticularly (PROLENE ${ }^{\mathrm{TM}}$, Ethicon Inc., United States).

\section{Results}

From 2011 to 2012, there were a total of five patients presented with strangulated groin hernia received single incision laparoscopic repair in our unit. Four of them were male and one of them was female, with the median age of 62 years (range 37 - 88 years old). Three of them suffered from strangulated indirect inguinal hernia while two of them suffered from strangulated femoral hernia. One of the patients who suffered from strangulated femoral hernia was also found to have concurrent bilateral inguinal hernia and they were repaired as well in the same operation. Two of them presented with clinical evidence of intestinal obstruction as shown on their abdominal X-ray. The median operative time was 75 minutes for unilateral repair (range 65 - 80 minutes). The operative time for the patient with strangulated unilateral femoral hernia and concurrent bilateral indirect inguinal hernia was 95 minutes. Two of the patients had small bowel loops as the content of the hernia while three of them had omentum as the content. One of the patient required resection of the infarcted omentum. None of the above patients required bowel resection. All single incision laparoscopic repairs were successfully performed. None of the patients required additional ports for conversion to conventional laparoscopic repair or open repair. The median hospital stay was 2 days (range 1 - 3 days). The median number of analgesics (tramadol $50 \mathrm{mg}$ tablet) required on the first post-operative day was 4 tablets. The mean of the overall wound satisfaction score was 8.6 out of 10 (range 8 - 10) on postoperative day 7 . There was no wound related complication in our series and none of the patients were found to have incisional hernia or recurrence of their groin hernia with a mean follow up of 12 months (range 8 - 18 months) (Tables 1 and 2).

\section{Discussion}

Traditionally open groin exploration with or without laparotomy was considered as the gold standard for management of patients presenting with strangulated groin hernia. Study had showed that first intention ex-

Table 1. Clinical details of patients undergoing SILS TAPP.

\begin{tabular}{cccccc}
\hline Sex & Age & $\begin{array}{c}\text { Duration of } \\
\text { operation (minutes) }\end{array}$ & $\begin{array}{c}\text { Type of } \\
\text { hernia }\end{array}$ & Content & $\begin{array}{c}\text { Viability of } \\
\text { content }\end{array}$ \\
\hline F & 66 & 75 & Femoral & Bowel & Yes \\
M & 62 & 65 & $\begin{array}{c}\text { Indirect } \\
\text { Inguinal }\end{array}$ & Bowel & Yes \\
M & 54 & 80 & $\begin{array}{c}\text { Indirect } \\
\text { Inguinal }\end{array}$ & Omentum & Yes \\
M & 37 & 75 & $\begin{array}{c}\text { Indirect } \\
\text { Inguinal }\end{array}$ & Omentum & Yes \\
M & 88 & 95 & Femoral & Omentum & No \\
\hline
\end{tabular}

Table 2. Intra-operative and post-operative details.

\begin{tabular}{lc}
\hline & Median/Mean (range) \\
\hline Operative time (minutes) & $75(65-80)$ \\
Need of bowel resection & 0 \\
Use of additional ports & 0 \\
Conversion to open & 0 \\
$\begin{array}{l}\text { Number of tablets of analgesics used } \\
\text { (tramadol } 50 \text { mg tablets) }\end{array}$ & $4(2-8)$ \\
Hospital stay (Days) & $2(1-3)$ \\
Mean of wound satisfaction score $(0-$ & $8.6(8-10)$ \\
$10,0=$ unsatisfied, $10=$ satisfied) & 0 \\
Wound infection & 0 \\
Recurrence of groin hernia & 0 \\
Umbilical hernia & \\
\hline
\end{tabular}


ploratory laparotomy in strangulated hernia surgery was a major cause of postoperative complication [4]. Laparoscopic groin hernia repair is now widely accepted and applied with the benefits of better cosmetic outcome, faster recovery and no difference in recurrence rate [1]. For patients with acutely strangulated groin hernia, emergency laparoscopic repair for strangulated groin hernias was shown to be feasible and appeared to have a lower morbidity relative to open repair as reported by Yang et al. [3]. With the use of laparoscopy, the strangulated part of the hernia could be reduced manually with external compression under direct laparoscopic view. Any intra-abdominal adhesions can be taken down under direct vision and thus facilitated the reduction. Reduction using laparoscopic instruments need to be extra cautious as it would easily result in serosal tear, especially in edematous bowel in emergency setting. Under most circumstances the strangulated content could be reduced by external pressure alone. In our series, none of the patient required extension of the hernia orifice for reduction of the hernia content. This might be explained by the stretching of the abdominal wall after the creation of pneumoperitoneum that dilated the hernia orifice. After reduction of the strangulated part, the hernia defect could be repaired. It also allowed enough time for the strangulated content to recover during the repair. Any concurrent hernia would also be identified during the laparoscopy. Some may argue that in case of strangulated hernia requiring bowel resection, the use of mesh for repair was contraindicated due to the risk of mesh infection. But recent studies had showed that bowel resection was not related to postoperative mesh infection nor wound infection [5].

Recent further advancement of laparoscopic surgery with single port instrument allowed further reduction of invasiveness of laparoscopic hernia repair. Subsequent reports had shown promising results of single incision laparoscopic repair in elective setting $[2,6]$. So far there was no published data about the use of single incision laparoscopy for patients presenting with acute strangulated groin hernia. To our knowledge, our series is the first to report on the use of SILS for this condition. In our series, a transumbilical approach was used. It was chosen because the $2 \mathrm{~cm}$ umbilical wound would easily allow small bowel to be bought through for resection if needed. It also offers a better cosmetic outcome since the scar is hidden inside the umbilicus (Figure 1). Also in patients with dilated and edematous bowel loops, working with single incision can avoid insertion of trocars in other part of the abdominal wall which can potentially lead to injury of the dilated bowel.

SILS was reported to have a higher pain score comparing to conventional laparoscopic repair. In this retrospective series, we assessed the level of pain according

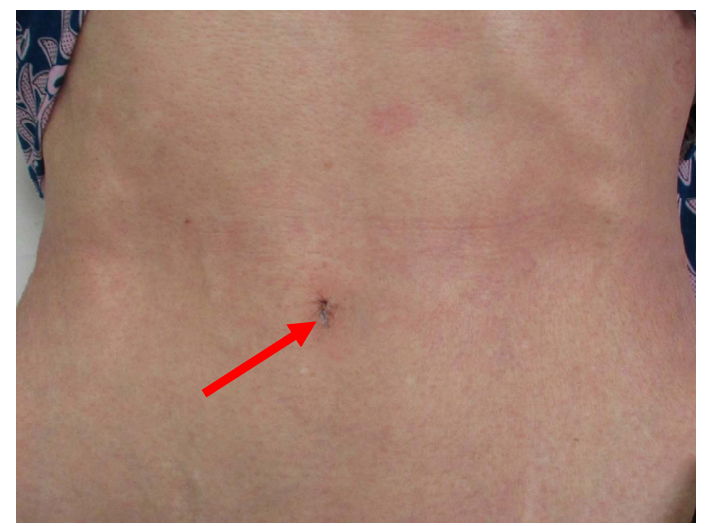

Figure 1. Umbilical wound (arrow) at 3 months post operatively.

to the number of analgesics that individual patient took. The median number of analgesics used was 4 tablets and the average satisfaction score of 8.6 out of 10 . This reflected that this technique was well accepted by our patients.

There were concerns about the risk of complication with umbilical hernia after SILS. Studies had shown that the risk of umbilical hernia was associated with obesity. The risk of occurrence was $0.5 \%$ versus $2.4 \%$ for patients with significant factors for fascial weakening [7]. In this series, none of the patient was found to have umbilical hernia upon follow-up. Similar result with no occurrence of umbilical hernia was also shown in our experience with 15 elective cases of single incision hernia repair performed in 2010 to 2011 [2]. In our unit, polydioxanone suture (PDS ${ }^{\mathrm{TM}}$ II, Ethicon) was used to close the fascial defect. Comparing to other types of absorbable suture, for example polyglactin suture (Coated VICRYL $^{\mathrm{TM}}$, Ethicon) which were commonly used by other centres, polydioxanone suture provided more durable strength retention. This might explain the low occurrence of umbilical hernia in our series.

The initial results of single incision laparoscopic repair for strangulated groin hernia were promising, although long term follow up data was lacking. No major complication was reported in our series. Technically single incision surgery had a steep learning curve. The loss of triangulation and the crowding effect of the instrument might obscure the laparoscopic view. In case of emergency when the bowels were dilated, it would further jeopardize the laparoscopic view. For better results, it was preferred to be performed in selected case by surgeons experienced in single incision laparoscopic surgery.

\section{REFERENCES}

[1] A. Grant, "Laparoscopic Compared with Open Methods of Groin Hernia Repair: Systematic Review of Random- 
ized Controlled Trials,” British Journal of Surgery, Vol. 87, No. 7, 2000, pp. 860-867. doi:10.1046/j.1365-2168.2000.01540.X

[2] G. P. C. Yang, E. C. H. Lai, O. C. Y. Chan, C. N. Tang and M. K. W. Li, "Single-Incision Transabdominal Preperitoneal and Totally Extraperitoneal Repair for Inguinal Hernia: Early Experience from a Single Center in Asia," Asian Journal of Endoscopic Surgery, Vol. 4, No. 4, 2011, pp. 166-170. doi:10.1111/j.1758-5910.2011.00098.x

[3] G. P. C. Yang, C. T. Y. Chan, E. C. H. Lai, O. C. Y. Chan, C. N. Tang and M. K. W. Li, "Laparoscopic versus Open Repair for Strangulated Groin Hernias: 188 Cases over 4 Years,” Asian Journal of Endoscopic Surgery, Vol. 5, No. 3, 2012, pp. 131-137. doi:10.1111/j.1758-5910.2012.00138.x

[4] B. Romain, R. Chemaly, N. Meyer, C. Brigand, J. P. Steinmetz and S. Rohr, "Prognostic Factors of Postoperative Morbidity and Mortality in Strangulated Groin Her- nia,” Hernia, Vol. 16, No. 4, 2012, pp. 405-410. doi:10.1007/s10029-012-0937-y

[5] J. Ueda, T. Nomura, J. Sasaki, K. Shigehara, K. Yamahatsu, A. Tani, Y. Shioda, K. Furukawa and E. Uchida, "Prosthetic Repair of an Incarcerated Groin Hernia with Small Intestinal Resection," Surgery Today, Vol. 42, No. 4, 2012, pp. 359-362. doi:10.1007/s00595-011-0019-2

[6] Y. Soon, E. Yip, S. Onida and H. Mangat, "Single-Port Hernia Repair: A Prospective Cohort of 102 Patients," Hernia, Vol. 16, No. 4, 2012, pp. 393-396. doi:10.1007/s10029-012-0917-2

[7] C. C. Gunderson, J. Knight, J. Ybanez-Morano, C. Ritter, P. F. Escobar, O. Ibeanu, F. C. Grumbine, M. A. Bedaiwy, W. W. Hurd and A. N. Fader, "The Risk of Umbilical Hernia and Other Complications with Laparoendoscopic Single-Site Surgery," Journal of Minimally Invasive Gynecology, Vol. 19, No. 1, 2012, pp. 40-45. doi:10.1016/j.jmig.2011.09.002 